\title{
Aporte al conocimiento de la conectividad del manglar de Las Lisas-La Barrona, Guatemala
}

\author{
Contribution to knowledge of mangrove connectivity of Las Lisas-La Barrona, Guatemala \\ Celia V. Dávila-Pérez ${ }^{1}$, Manolo J. García², Pilar M. Velásquez-Jofre² \\ ${ }^{1}$ Centro de Estudios del Mar y Acuicultura, ${ }^{2}$ Centro de Estudios Conservacionistas, \\ ${ }^{3}$ Facultad de Cienicas Químicas y Farmacia, Universidad de San Carlos de Guatemala, Guatemala
}

*Autor al que se dirige la correspondencia:

Recibido: 09 de octubre 2018 / 1era. Revisión: 18 de marzo 2019 / 2da. Revisión: 23 de septiembre 2019 / Aceptado: 31 de octubre 2019

\section{Resumen}

Ql presente estudio es una primera aproximación al conocimiento de la conectividad del ecosistema manglar - $d$ de Las Lisas-La Barrona, departamentos de Santa Rosa y Jutiapa. La conectividad es la característica del paisaje que facilita la dispersión de las especies a través del hábitat, la pérdida de esta conectividad, podría poner en peligro la sobrevivencia de las poblaciones de aves. El objetivo de este estudio fue determinar la conectividad del manglar a través de análisis basados en las teorías de grafos y circuitos. Se incluyó en el análisis la conectividad en sus componentes estructural y funcional. El componente estructural se determinó mediante el Análisis del Patrón Espacial Morfológico (MSPA por sus siglas en inglés). El componente funcional fue evaluado con el Diferencial del êndice Integral de Conectividad (dIIC), evaluando el aporte de cada parche a la conectividad del manglar. Se encontró que la conectividad del área está determinada por parches nœcleo con alto aporte a la conectividad como los parches de vegetación de mangle de La Barrona y El Jiote, parche puente o corredor como el canal de Chiquimulilla y un cuello de botella en el segmento Las Lisas-El ahumado, los cuales son elementos estratégicos a considerar en la planificación e intervenciones futuras en el área.

Palabras claves: paisaje, costa del Pacífico, Guidos, Conefor, CircuitScape

\begin{abstract}
The present study is a first approach to the knowledge of the connectivity of the mangrove ecosystem of Las 1 Lisas-La Barrona. Connectivity is the landscape characteristic that facilitates the dispersion of species through habitat, the loss of this connectivity, could endanger the survival of bird populations. The objective was to estimate mangrove connectivity through analysis based on the graphs and circuits theories. The connectivity was estimated on its structural and functional components. The structural component was determined by a Morphological Spatial Pattern Analysis (MSPA). The functional component was assessed with the Differential of the Integral Connectivity Index (dIIC), evaluating the contribution of each patch to the mangrove connectivity. It was found that the connectivity of the area is determined by cores patches with high contribution to connectivity such as the mangrove vegetation patches of La Barrona and El Jiote, bridges or corridors such as the canal de Chiquimulilla and a bottleneck in the Las Lisas - El Ahumado segment, which are strategic elements to consider in planning and future interventions in the area.
\end{abstract}

Keywords: landscape, Pacific coast, Guidos, Conefor, CircuitScape 


\section{Introducción}

A nivel global, la diversidad biológica marino costera, ha sido identificada como elemento estratégico del desarrollo humano por los servicios ecosistémicos que provee (Costanza et al., 1997). Sin embargo, esta diversidad está siendo afectada en su estructura y funcionamiento por una variedad de actividades humanas (van den Belt \& Cole, 2014).

La conectividad se puede definir como el movimiento de organismos a través de los parches de hábitats de un paisaje (Olds, Pitt, Maxwell, \& Connolly, 2012). Los dos componentes de la conectividad son la conectividad estructural que está basada en la forma y disposición de los parches de un hábitat, y la funcional que se basa en la respuesta conductual de las especies a la estructura física del paisaje (Bennett, 1998).

Existen métodos para identificar los parches más importantes para mantener la conectividad en las redes de hábitat (Pascual \& Saura, 2008; Saura \& Rubio, 2010). Se considera que estos métodos de priorización son útiles para favorecer una mejor asignación de fondos y esfuerzos para proteger la diversidad biológica, y ayudar a definir las medidas de conservación, restauración y mitigación (Pascual \& Saura, 2008).

En cuanto a la conectividad estructural uno de los análisis utilizados es el Análisis del Patrón Espacial Morfológico (MSPA), el cual es útil para identificar elementos del paisaje de mayor relevancia para mantener la conectividad de acuerdo a su forma y disposición (European Comission, 2018). En el componente funcional donde influyen factores como los desplazamientos de dispersión de las especies, es útil la estimación de índices de conectividad como el Diferencial del Índice Integral de Conectividad (dIIC) el cual estima la contribución de cada parche a la conectividad del sistema en sus tres fracciones: (a) dIIC-interna, que es la contribución de un parche a la conectividad interna del sistema; (b) dIIC-flujo que corresponde al flujo de dispersión ponderado por área a través de las conexiones de un parche determinado (c) dIIC-conector que es la contribución de un parche determinado a la conectividad, fungiendo como escalón hacia otros parches (Saura \& Rubio, 2010).

Tomando en cuenta que la conectividad de los bosques debe considerarse como uno de los objetivos prioritarios de los planes de conservación y planificación de los ecosistemas (Pascual \& Saura, 2008), esta investigación tuvo como objetivo estimar la conectividad estructural y funcional del ecosistema manglar de Las Lisas-La Barrona.
Este humedal está propuesto para ser designado como área protegida y sitio Ramsar (Consejo Nacional de Áreas Protegidas [CONAP] \& Programa de las Naciones Unidas para el Desarrollo [PNUD], 2017), y es uno de los sitios clave para la conservación de aves acuáticas en Guatemala (Eisermann \& Avendaño, 2007). El proceso de planificación y construcción de las herramientas de gestión y manejo del área demanda contar con criterios que permitan considerar los elementos estratégicos de la conectividad del ecosistema manglar. Por lo que el conocimiento generado en esta investigación puede orientar la toma de decisiones en la planificación espacial del área.

\section{Materiales y métodos}

\section{Área de estudio}

El humedal costero de Las Lisas-La Barrona se encuentra localizado en el litoral del Pacífico de Guatemala, en los departamentos de Santa Rosa y Jutiapa. Se caracteriza por diversos ecosistemas y áreas de importancia ecológica como los manglares, playas arenosas, playas fangosas, dunas, bocabarras y una sección de aproximadamente $15 \mathrm{~km}$ del canal de Chiquimulilla, el cual es un canal estuarino. Se localiza en las coordenadas GTM Datum WGS 84 longitud 788635.4689 y latitud 1530421.39 , longitud 788865.0561 y latitud 1531052.755 , longitud 789926.897 y latitud 1531024.057 , longitud 791534.0075 y latitud 1530220.501 (Conap \& PNUD, 2017) (Figura 1).

Tiene un área de 4,116.53 ha, dentro de la cual hay 1,405.89 ha de ecosistema manglar que está caracterizado por la presencia de al menos cuatro especies de manglares Rhizophora mangle L. (mangle rojo), Laguncularia racemosa (L.) Gaertn. (mangle blanco), Avicennia germinans (L.) L. (mangle negro) y Conocarpus erectus L. (botoncillo) (Conap \& PNUD, 2017). Se localiza en la zona de vida denominada bosque seco tropical (bs-T), presenta temperaturas promedio anuales por encima de los $24^{\circ} \mathrm{C}$, los rangos de precipitación pluvial promedio anual van de 700 a $1,850 \mathrm{~mm}$ (Instituto de Investigación y Proyección sobre Ambiente Natural y Sociedad de la Universidad Rafael Landívar [Iarna-URL], 2018).

\section{Procedimiento}

Se realizó la estimación de la conectividad del manglar del área de estudio, a través de análisis basados 

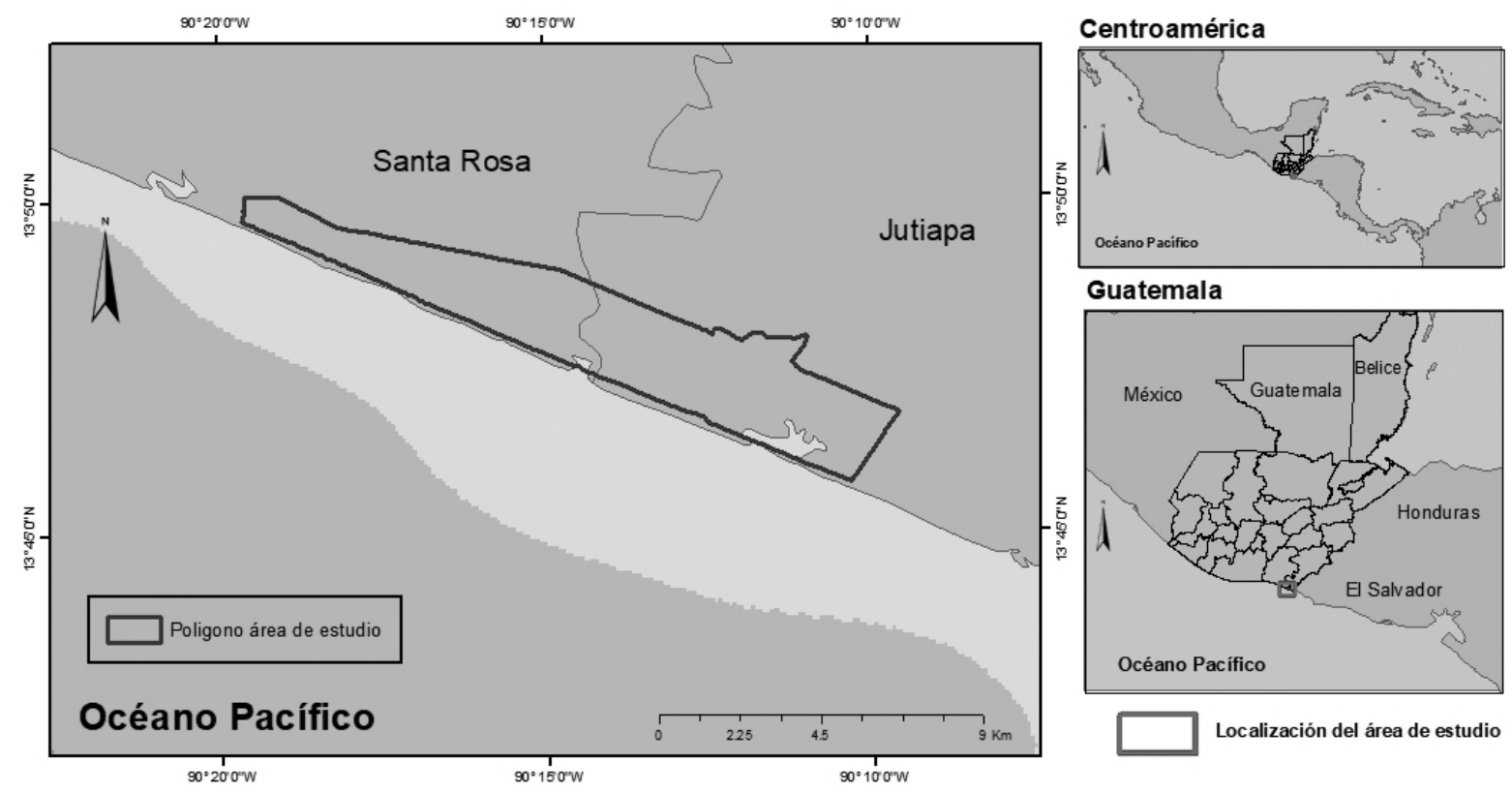

Figura 1. Mapa de ubicación del humedal Las Lisas-La Barrona.

en teoría de grafos y circuitos. Las aplicaciones de la teoría de grafos a la ecología del paisaje han mostrado el potencial para cuantificar la conectividad del paisaje para una especie o grupo de especies, centrándose en el papel de los parches de hábitat y los vínculos de dispersión entre ellos (Saura, \& Rubio, 2010).

La teoría de circuitos se ha aplicado a la conectividad en análisis químicos, neurales, económicos y sociales. Es viable para la ecología, donde se utilizan métricas basadas en conectividad eléctrica que son aplicables a la ecología del movimiento, proporcionando interpretaciones ecológicas concretas del circuito-teórico (McRae, Dickson, Keitt, \& Shah, 2008).

Para los procedimientos espaciales se utilizó el programa ArcMap versión 10 (Environmental Systems Research Institute [ESRI], 2016) licencia de ArcMap Desktop del Centro de Datos para la Conservación del Centro de Estudios Conservacionistas (Cecon). Conjuntamente se utilizaron diversos softwares libres para la aplicación de los análisis espaciales de la conectividad, los cuales se mencionan a continuación.

\section{Análisis de conectividad estructural}

Se utilizó el cálculo del MSPA, el cual analiza la forma y disposición de los parches de un hábitat, y puede detectar los sitios del paisaje de mayor relevancia para mantener la conectividad de acuerdo a su morfología (Soille \& Vogt, 2008).

Se generó el mapa de uso y cobertura del suelo del área de estudio (Figura 2) a partir de la validación y corroboración en campo mediante dos visitas al área de estudio durante los meses de septiembre (20-21) y noviembre (21-25) del 2016. Finalmente a partir de la imagen del satélite Sentinel 2, Sentinel Scientific Data Hub de la Agencia Espacial Europea (ESA) $(02 / 02 / 2017)$ se obtuvo el mapa de uso del suelo del área de estudio a partir del cual se generó un mapa binario de cobertura de mangle en formato raster.

A este mapa se aplicó el MSPA, con el software libre GuidosToolbox2.7 (Vogt \& Riitters, 2017). Este análisis estimó la forma y disposición espacial de los parches de manglar, clasificándolos por su morfología en: (a) núcleo: área interior excluyendo el perímetro, (b) borde: perímetro externo del objeto, (c) puente: conectado a diferentes áreas del núcleo, (d) isla: islote disjunto y demasiado pequeño para contener núcleo, (e) perforación: perímetro interno del objeto, (f) lazo: conectado a la misma área del núcleo y (g) rama: conectando los núcleos con parches adyacentes. 


\section{Análisis de conectividad funcional potencial}

Estimación del diferencial del Índice Integral de Conectividad (dIIC). Se estimó el diferencial del Índice Integral de Conectividad (dIIC) el cual es un índice que va de 0 a 1 y aumenta con la conectividad mejorada. $\mathrm{El}$ IIC $=1$ en el caso hipotético de que todos los parches del paisaje están ocupados por el hábitat. Este permite conocer el aporte de cada parche de manglar en la conectividad de todo el sistema, para lo cual se utilizó el software libre Conefor Sensinode versión 2.2 (Pascual \& Saura, 2008; Saura \& Torné, 2009). De manera complementaria se aplicó un análisis de flujo de corriente acumulada (McRae, Shah, \& Mohapatra, 2013).

Este análisis se corrió a partir de los núcleos obtenidos del MSPA y el canal de Chiquimulilla. Se emplearon las aves como especies focales, para lo cual, mediante revisión bibliográfica se generó un listado de especies de aves para el área de estudio (Conap \& PNUD, 2017; Dávila, García, \& López, 2014; Eisermann \& Avendaño, 2007; Salazar \& Sigüenza, 2010). Las especies se priorizaron con base a su estatus residente y su estado de conservación (International Union for Conservation of Nature [IUCN], 2018), posteriormente se agruparon con base en los grupos funcionales de aves de acuerdo a Dávila y colaboradores (2014) y se asignaron los valores de 5 y $10 \mathrm{~km}$ como valores de distancias de dispersión por grupo funcional, de acuerdo a National Council for Air and Stream Improvement (NCASI) (2004), cuyos valores se describen dentro de las especificaciones de ocurrencia del elemento para los grupos de aves.

Estimación del flujo de corriente acumulada. Esta estimación se basa en la teoría de circuitos, los modelos de circuitos se pueden usar para predecir patrones de movimiento en paisajes complejos e identificar parches importantes de hábitat para la planificación de la conservación. Los modelos de conectividad basados en teoría de circuitos, brindan ventajas sobre los modelos de conectividad. La resistencia, la corriente y el voltaje calculados a través de gráficos pueden interpretarse y relacionarse con procesos ecológicos, tales como el movimiento individual de especies en un mosaico de hábitats (McRae et al., 2008; Pelletier et al., 2014).

Se realizó bajo el supuesto de que la resistencia del paisaje es una medida de cuán fácil un organismo puede migrar o dispersarse a través de los elementos del paisaje (Colorado, Vásquez, \& Mazo, 2017; Rose, 2013). En este análisis se utilizaron los parches identificados como núcleos con el MSPA y un mapa de resistencia.

El mapa de resistencia, se originó a partir de catalogar los tipos de uso y cobertura del suelo como barreras, bajo el supuesto de que una barrera es toda aquella limitación que en la matriz del paisaje presenta una resistencia para el desplazamiento de las aves (Bartesaghi, 2015).

De acuerdo a los criterios cuantitativos de Bartesaghi (2015), Colorado y colaboradores (2017) y Desrochers, Bélisle, Morand-Ferron y Bourque (2011), los valores se modificaron y se simularon las barreras de movimiento en la dispersión de las aves, cuando la resistencia al movimiento en áreas abiertas varió de 1 a 100 veces con respecto a la resistencia al movimiento en el bosque de manglar. Con lo que se designaron los siguientes valores a las barreras con respecto al movimiento de las aves: (1) a los ambientes con mayor intensidad de uso (bosque, espejo de agua), (4) ambientes de uso frecuente (arena, playa, estanques de acuicultura, salineras), (11) a los ambientes de uso ocasional (campos de agricultura o ganadería), (100) a las barreras de movimiento (áreas urbanas, áreas con infraestructura). Consecutivamente se aplicaron los criterios a los diferentes tipos de cobertura y usos del suelo, asignándoles valores de resistencia al movimiento (Tabla 1). Finalmente, con los núcleos del MSPA y el mapa de resistencia, se efectuó el análisis de flujo de corriente acumulada, para lo cual se utilizó el software libre Circuit Scape 4.0 (McRae et al., 2013).

\section{Resultados}

\section{Estimación de la conectividad estructural}

El ecosistema manglar de Las Lisas-La Barrona presenta una conformación espacial definida por diferentes tipos de parches. El MSPA mostró que los tres tipos morfológicos de parches con mayores porcentajes son los núcleos, bordes y perforaciones. La clase de parche mejor representada en el área con respecto al porcentaje de cobertura son los núcleos (80.94\%), encontrándose principalmente en La Barrona, El Jiote y, con menor representatividad en Las Lisas.

Posteriormente se ubican los bordes (11.66 \%), las perforaciones $(3.59 \%)$, las ramas $(1.49 \%)$, que se encuentran principalmente cerca de El Chapetón, El Ahumado y Las Lisas; los lazos (0.99\%), que se 


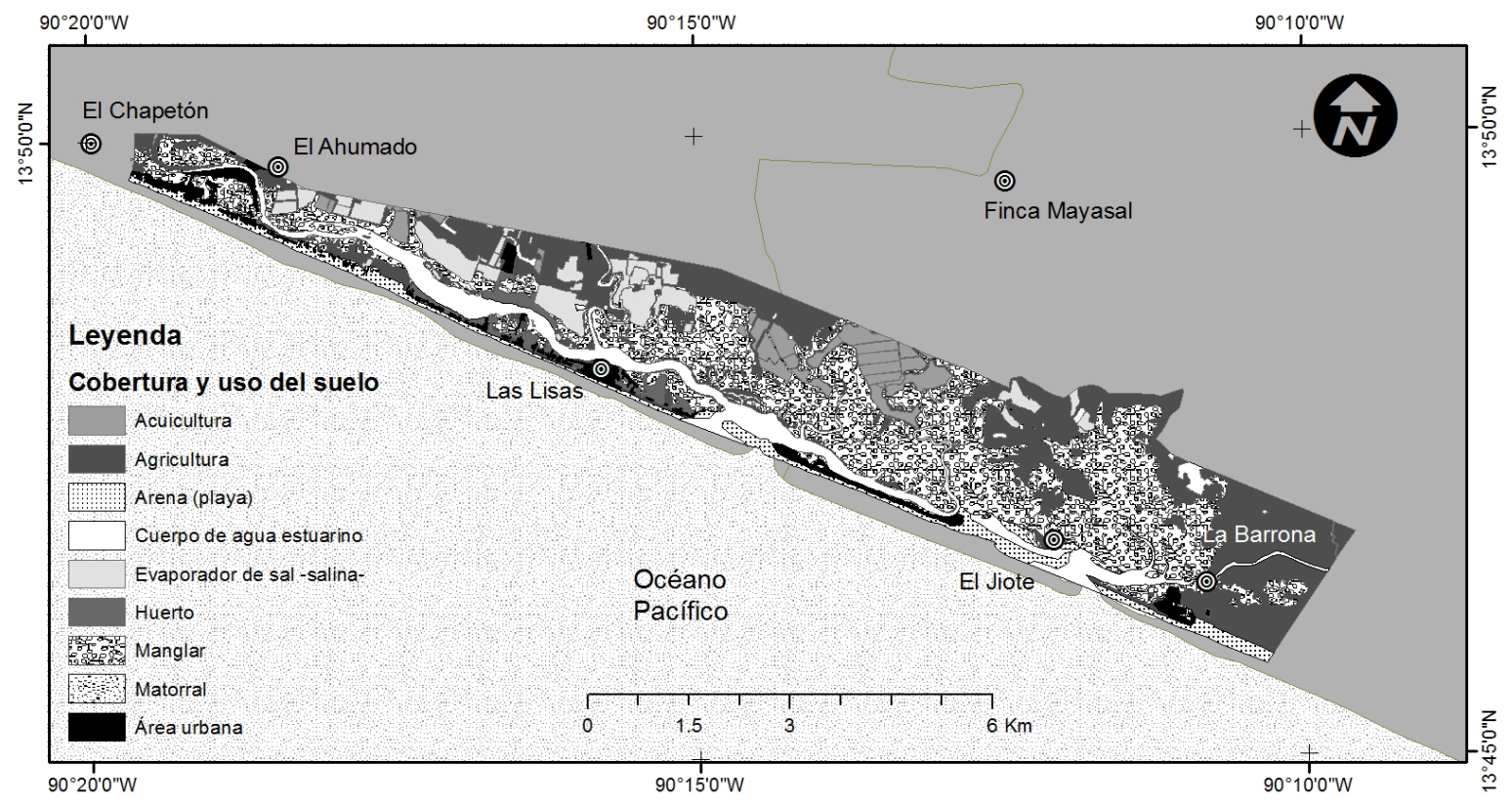

Figura 2. Mapa de la cobertura y uso del suelo en el humedal Las Lisas-La Barrona.

Tabla 1

Tipo de Cobertura y usos del suelo, y asignación de valores de resistencia

\begin{tabular}{clcc}
\hline No. & \multicolumn{1}{c}{ Tipo de cobertura y usos del suelo } & Área (ha) & Valores de resistencia \\
\hline 1 & Manglar & $1,405.89$ & 1 \\
2 & Agricultura & $1,022.70$ & 11 \\
3 & Cuerpo de agua estuarino & 390.88 & 1 \\
4 & Salina (evaporador de agua marina) & 222.79 & 4 \\
5 & Arena (playa) & 182.39 & 4 \\
6 & Acuicultura & 174.14 & 4 \\
7 & Área urbana o área con infraestructura & 127.96 & 100 \\
8 & Huerto & 65.20 & 4 \\
9 & Matorral & 14.58 & 4 \\
\hline
\end{tabular}

encuentran conectado a la misma área de los núcleos de La Barrona, El Jiote y Las Lisas; los puentes (0.81 \%), que están presentes en Las Lisas, El Ahumado y El Chapetón; y los parches tipo isla $(0.52 \%)$, que sobresalen en El Ahumado y El Jiote (Tabla 2, Figura 3).

\section{Estimación de la conectividad funcional potencial}

Estimación del dIIC. Se detectaron 153 especies de aves, de las cuales se priorizaron 96, las cuales se agruparon en 20 grupos con base en los grupos funcionales de aves. Los resultados mostraron que la 


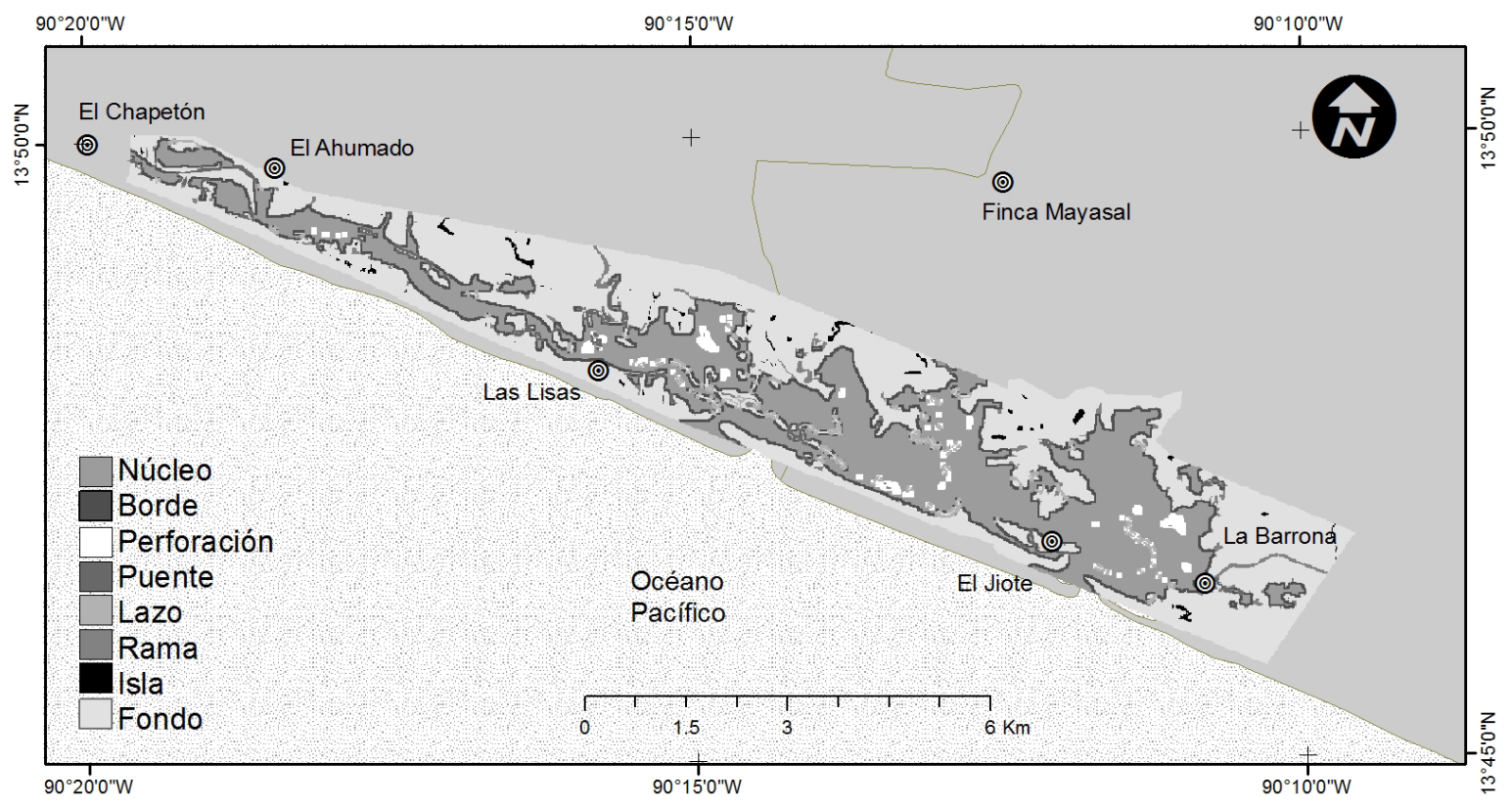

Figura 3. Patrón morfológico espacial del ecosistema manglar del área de estudio.

Tabla 2

Porcentaje de cada clase del tipo de parche de acuerdo al cálculo del MSPA

\begin{tabular}{lccc}
\hline Clase & \% de la cobertura de mangle & \% del área analizada & Frecuencia \\
\hline Nœcleo & 80.94 & 40.36 & 81 \\
Borde & 11.66 & 5.82 & 143 \\
Perforaciones & 3.59 & 1.79 & 138 \\
Rama & 1.49 & 0.74 & 301 \\
Lazo & 0.99 & 0.49 & 129 \\
Puente & 0.81 & 0.4 & 68 \\
Isla & 0.52 & 0.26 & 54 \\
\hline
\end{tabular}

conectividad tiene un gradiente que va en aumento de oeste a este en el área de estudio, mostrando mayores valores de la estimación del dIIC en la Barrona y el canal de Chiquimulilla, seguidos por El Jiote y con valores menores en Las Lisas.

El cálculo del dIIC mostró que los parches con mayor valor del dIIC son La Barrona y el canal de Chiquimulilla siendo las áreas con mayor importancia en el mantenimiento de la conectividad del área tanto para aves que se dispersan $5 \mathrm{~km}$ (Figura 4), como para aquellas que se dispersan $10 \mathrm{~km}$ dentro del área de estudio (Figura 5).
Las áreas con mayor dIIC interno se localizan desde Las Lisas en Santa Rosa hacia el área de La Barrona en Moyuta, Jutiapa, presentando los valores más altos hacia La Barrona, tanto en el análisis para $5 \mathrm{~km}$ como en el análisis para $10 \mathrm{~km}$ de dispersión de las aves.

El dIIC-flujo es mayor en el área de La Barrona, lo cual disminuye hacia Las Lisas y se observa este patrón de disminución de la conectividad flujo hacia el área de El Ahumado, tanto para el análisis aplicado a especies que se dispersan $5 \mathrm{~km}$ como para las aves que se dispersan $10 \mathrm{~km}$. 


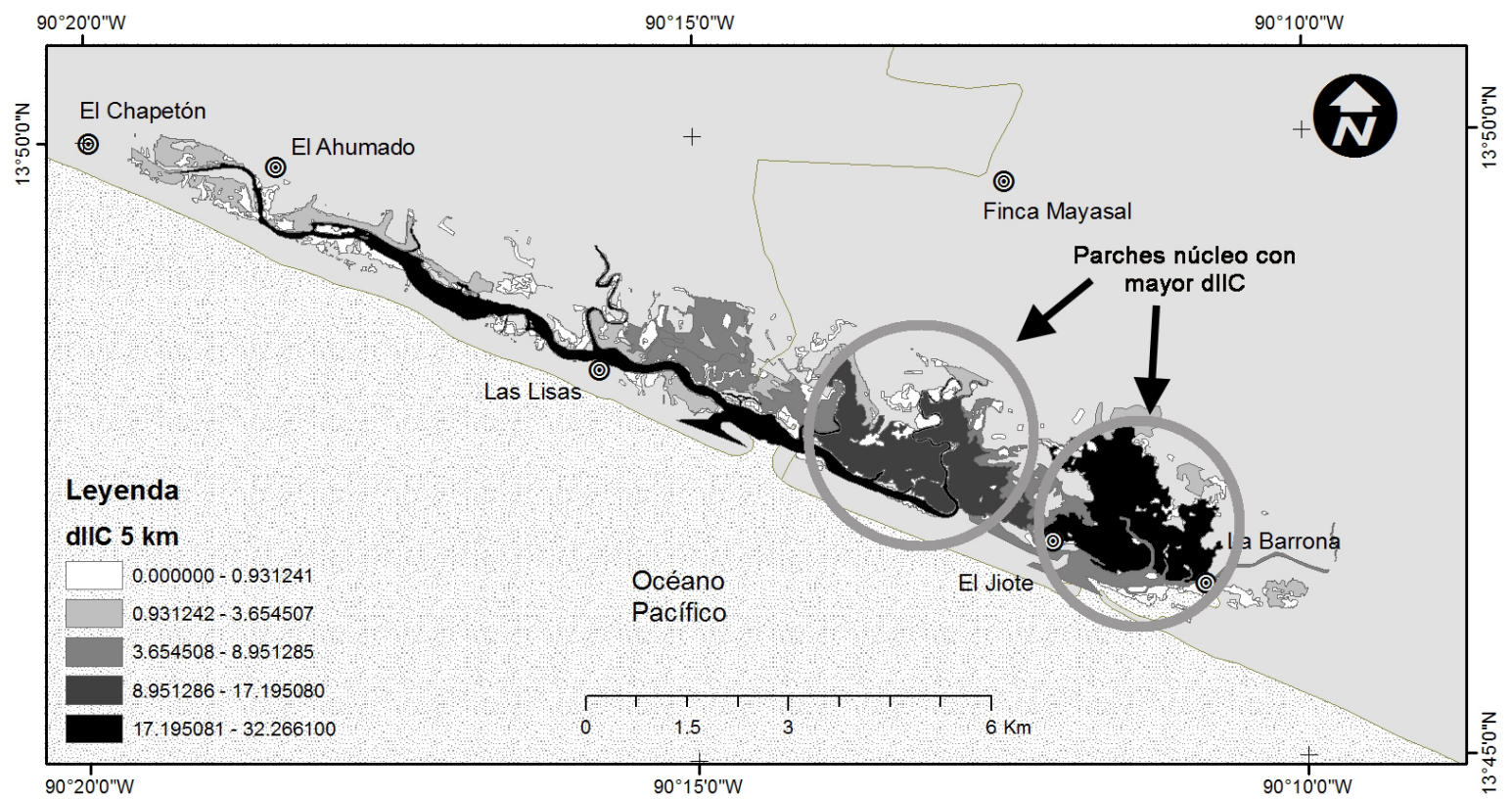

Figura 4. Mapas sobre el valor del dIIC (interno, flujo y conector) del manglar para especies que se dispersan $5 \mathrm{~km}$. El color blanco es la menor conectividad, al aumentar la tonalidad de gris a negro aumenta la conectividad. En el círculo gris se indican los parches con mayor dIIC.

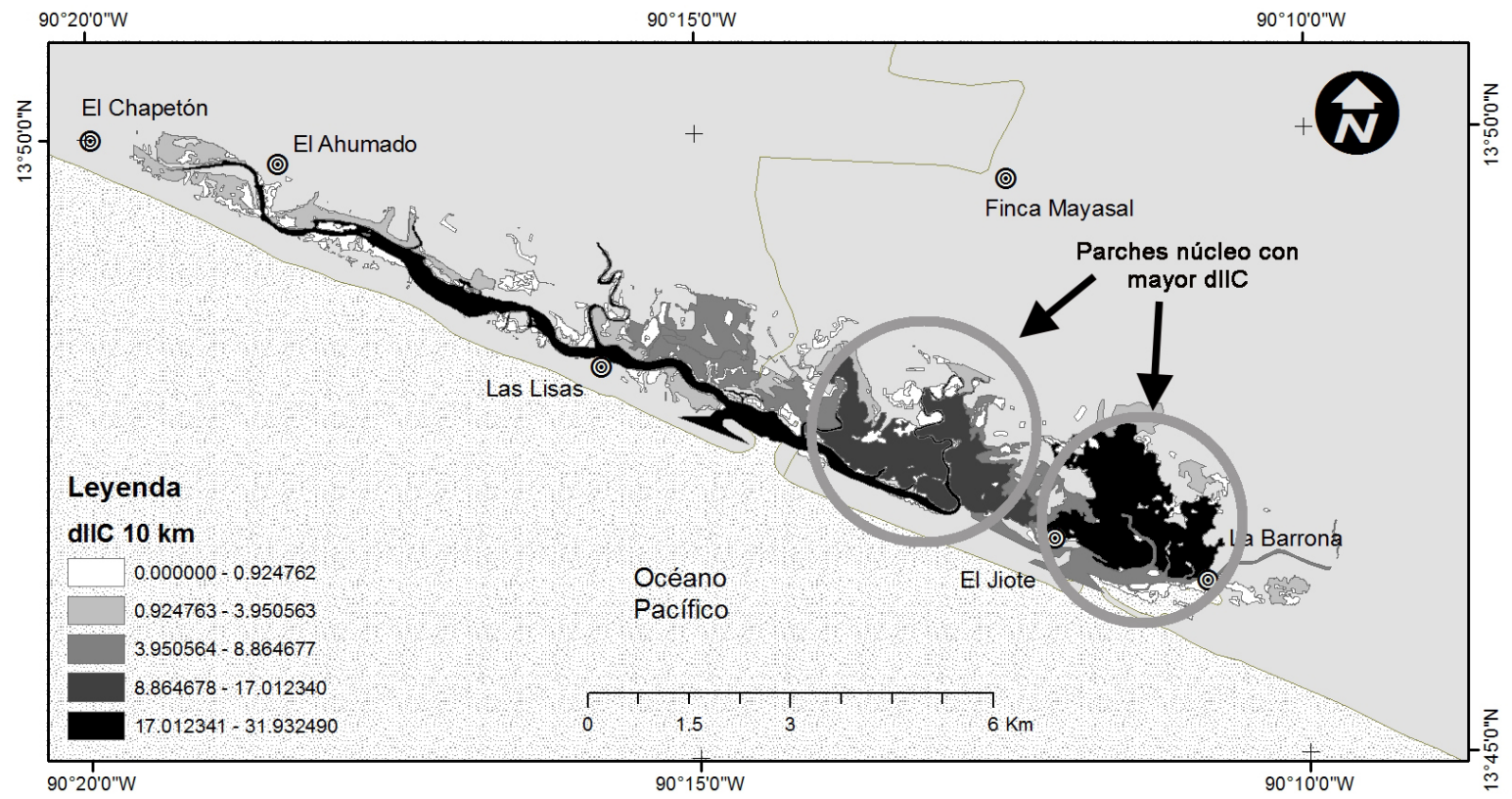

Figura 5. Mapa sobre el valor del dIIC (interno, flujo y conector) del manglar para especies que se dispersan $10 \mathrm{~km}$. El color blanco es la menor conectividad, al aumentar la tonalidad de gris a negro aumenta la conectividad. En el círculo gris se indican los parches con mayor dIIC. 
El dIIC-conector presentó que las áreas desde Las Lisas en Santa Rosa hacia el área de La Barrona en Moyuta, Jutiapa con mayor dIIC son las que aportan mayor conectividad, presentando los valores más altos hacia La Barrona, esto se observó en el análisis para 5 km de dispersión de las aves (Figura 6), pero no es así en el análisis para $10 \mathrm{~km}$ de dispersión.

Estimación del flujo de corriente acumulada. La estimación indicó que existen diferentes categorías de flujo de corriente acumulada en el ecosistema manglar. En particular, para el área se observa un "cuello de botella" localizado justo entre las localidades El Ahumado y Las Lisas. De acuerdo a la teoría de circuitos poseen el mayor paso de electricidad, lo que se puede interpretar como un sitio donde existe un alto flujo de movimiento de especies (Figura 7).

\section{Discusión}

\section{Conectividad estructural}

En la actualidad el ecosistema manglar ocupa el $40.36 \%$ del área de estudio, y los resultados del MSPA indican que aproximadamente el $81 \%$ de esta cobertura corresponde al tipo núcleo. Esta disposición espacial de los parches de manglar puede ser beneficiosa para la conservación del ecosistema, debido a que se presenta un menor efecto de borde de las presiones externas y una mayor capacidad de recuperación ante las mismas, manteniendo poblaciones con menor riesgo de extinción (Alonzo-F., Finegan, Brenes, Gunter, \& Palomeque, 2017; Gandini \& Lara, 2012).

Sin embargo, los parches tipo borde se ubican en segundo lugar en cuanto a porcentaje de cobertura, lo cual podría indicar que ha ocurrido cierto grado de fragmentación del ecosistema (Hernando, Velázquez, Rosario, Pérez, \& Grande, 2013). En este sentido, se recomienda evitar que ocurran modificaciones en la disposición espacial del manglar a través de la deforestación u otros tipos de intervenciones que conduzcan a la disminución de los parches tipo núcleo.

A pesar de que los puentes únicamente representan el $1 \%$ de la cobertura de manglar, este tipo de parches es estratégico, dadas las posibles ventajas que pueden proveer los puentes como corredores, entre ellas, facilitar el movimiento de especies y material genético a través del paisaje, aumento de la inmigración a hábitats aislados, facilitación de la continuidad de los procesos ecológicos naturales en hábitats impactados por las actividades humanas, entre otros (MacDonald, 2003). Por lo anterior, es importante resaltar y sugerir la priorización como elementos a conservar y aplicar medidas para mejorar la condición en la planificación espacial del área.

Los parches tipo lazo y rama, son elementos lineales del paisaje de poca anchura, pero que al mismo tiempo pueden ser el último vestigio de un bosque, siendo un importante refugio para la fauna. Este tipo de parches deben ser atendidos y restaurados, por el papel que pueden desempeñar en la conectividad y porque además tienen un elevado potencial de riesgo de alteración producido por la modificación del hábitats y pérdida de conectividad (Calabuig, 2013).

Este tipo de parches que han sido descritos en otros estudios, como en el caso de los corredores de cercos vivos, que se asemejan mucho a los parches lazo y rama del área de estudio, han sido el objetivo de estudio para el trazado de corredores biológicos, ya que estos pueden reducir los efectos de la deforestación y el desarrollo de infraestructura, fungiendo como piezas elementales para la dispersión de especies de aves y otros grupos taxonómicos (Calabuig, 2013).

Por otro lado, los parches tipo isla aunque presentan solo $0.52 \%$, toman relevancia al ser elementos útiles para aplicar técnicas de regeneración del ecosistema en áreas degradadas, una técnica muy común que se conoce como nucleación. La nucleación constituye una alternativa viable para la recuperación de los paisajes fragmentados, y será efectivo en el paisaje a restaurar, principalmente hacia las áreas desconectadas por la fragmentación (Tres \& Reis, 2007).

\section{Conectividad funcional potencial}

Estimación del dIIC. Los resultados encontrados concuerdan con lo hallado en la conectividad estructural, debido a que algunos de los parches con mayor dIIC corresponden al tipo núcleo, tales como los parches de La Barrona y El Jiote por lo que se puede deducir que son los parches con mayores potenciales para mantener la conectividad. Resaltando nuevamente el importante papel que pueden desempeñar estos en el mantenimiento de poblaciones con menor riesgo de extinción a través de una mayor conectividad interna del ecosistema manglar. Por lo tanto, se debe priorizar su conservación en la planificación territorial del área de estudio. Así mismo, en los resultados se evidencia la 


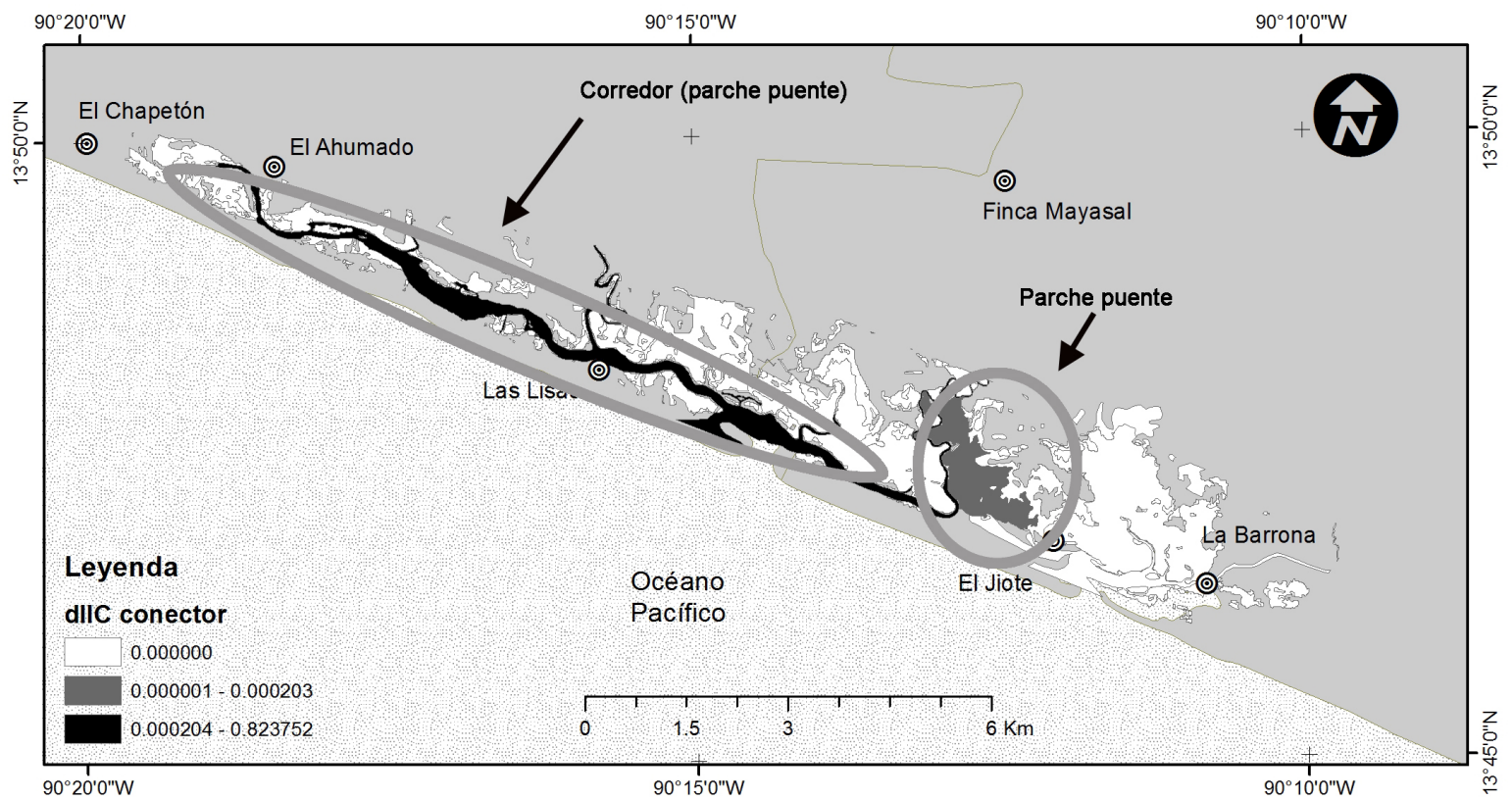

Figura 6. Mapa sobre el valor del dIIC (conector) del ecosistema manglar para especies que se dispersan $5 \mathrm{~km}$. El color blanco es la menor conectividad, al aumentar la tonalidad de gris a negro aumenta la conectividad. En el círculo gris se indican los parches con mayor dIIC.

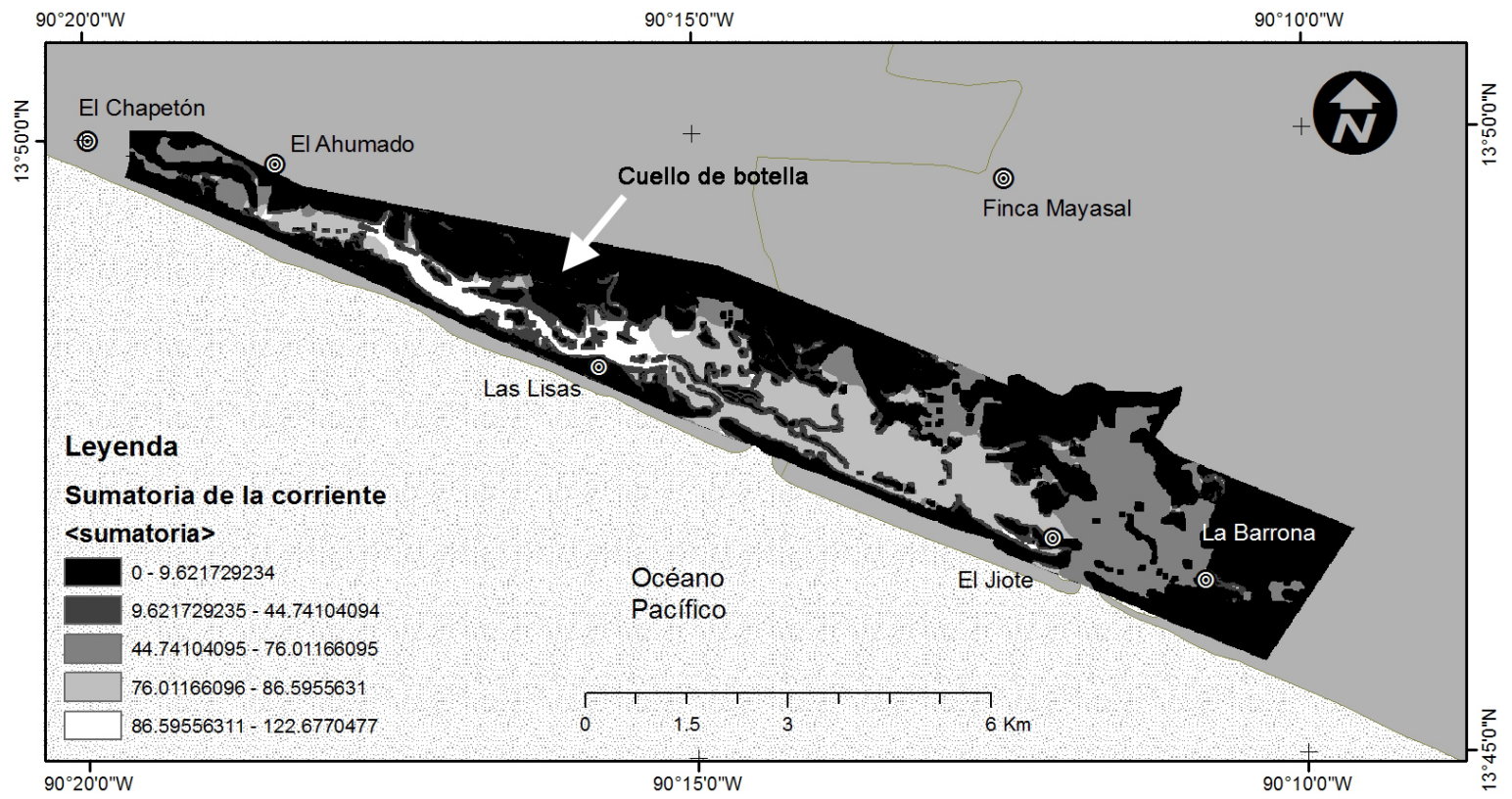

Figura 7. Mapa de la estimación del flujo de corriente acumulada del ecosistema manglar. Descripción: los menores valores de corriente acumulada se presentan en tonalidades negras y grises, aumenta a partir del gris claro y el color blanco posee el mayor valor de corriente acumulada (segmento cuello de botella). 
relevancia del canal de Chiquimulilla como elemento conector del ecosistema manglar.

Estos resultados concuerdan con Shanthala, Murthy, Debnath y Jha (2016), quienes estimaron el IIC en el paisaje forestal del distrito de East Godavari, India, donde obtuvieron que los parches núcleo tienen mayor potencial para la conectividad, por su alto valor de ponderación de IIC del parche más grande, a diferencia de los parches borde, con un bajo valor de ponderación de IIC.

Para el presente estudio también es relevante el hecho de que la fracción dIIC-flujo tiene una importante contribución a la conectividad del manglar, considerando la relevancia de asegurar la conectividad a una mayor escala, la conectividad de flujo puede conectar a este humedal con los manglares adyacentes, como los presentes en el Parque Nacional Hawaii al oeste y hacia el este con humedales del departamento de Ahuachapán, en El Salvador.

Cordero (2014) encontró en el hábitat potencial del mono aullador negro o carayá (Alouatta caraya), en un sistema silvopastoril del chaco en Paraguay, que la fracción dIIC-flujo presentó la mayor ponderación de IIC, lo que sugirió que muchos parches facilitan el flujo como fuente y receptor para la dispersión del carayá que tiene una dispersión de $0.84 \mathrm{~km}$.

En el presente estudio, la fracción dIIC-interna ponderó que los núcleos La Barrona y El Jiote, presentaron los mayores valores de conectividad, lo que tiene una relación directa con el mayor tamaño de estos parches. Lo cual concuerda con lo que encontró Cordero (2014), en el hábitat del carayá, donde los parches que presentaron los mayores valores de la fracción interna fueron los parches de bosque con la mayor área.

En esta comparación se hace con grupos diferentes de fauna, para aves y para un mamífero arborícola, sin embargo, resalta por el hecho de que sugiere que los parches núcleo de mayor área aportan la mayor conectividad al sistema estudiado, con lo que se puede inferir que pueden sostener poblaciones de aves y mamíferos con menor riesgo de extinción y ser fuente de especies, asumiendo el rol de continente como lo señala Alonzo-F y colaboradores (2017). Lo anterior alude a la importancia de conservar estos parches núcleo con altos valores de ponderación de IIC.

Por su parte, la fracción dIIC-conector ponderó a un menor número de parches, indicando que son pocos los parches en disposición de ejercer como conectores o parches puente para especies de aves que se dispersan 5 $\mathrm{km}$. En este caso el canal de Chiquimulilla y Las Lisas resultan ser los elementos del ecosistema manglar más importantes como elemento conector o parches puente. Lo anterior también se observó en los resultados de Cordero (2014) que encontró un bajo número de parches que aportan la conectividad conector, pero que fungen como parches puente de gran importancia para la conectividad en el hábitat del carayá.

Esto sugiere que el canal de Chiquimulilla es relevante como parche puente o incluso como corredor, que permite el desplazamiento de especies de aves que se dispersan $5 \mathrm{~km}$. Los efectos de los corredores para las aves varían según el área y no están bien documentados. Sin embargo, la estructura de escalones o parches bastante cercanos entre sí formando elementos lineales como corredores parecería ser una buena opción para facilitar la dispersión y la conectividad en un paisaje (Calabuig, 2013). Para especies que se dispersan 10 $\mathrm{km}$ la fracción dIIC-conector no muestra una porción importante, lo cual se debe a que la dispersión de 10 $\mathrm{km}$, probablemente utilizará al área como un conector entre humedales adyacentes.

Estimación del flujo de corriente acumulada. A este respecto, el sitio más crítico para la conectividad se encuentra justo entre las localidades El Ahumado y Las Lisas, ya que presenta el mayor valor de corriente acumulada para especies de aves que se dispersan 5 y 10 $\mathrm{km}$, siendo un cuello de botella. De acuerdo a Pelletier y colaboradores (2014) estos sectores poseen el mayor paso de electricidad, lo que se puede interpretar como que existe un alto flujo de movimiento de especies.

Por su parte Bartesaghi (2015) identificó zonas de cuellos de botella en la cuenca alta atlántica de los departamentos de Rocha y Maldonado, Uruguay, al evaluar el flujo de corriente acumulada para especies de grupos taxonómicos como moluscos, anfibios, reptiles, mamíferos y aves, con un rango de dispersión de 1 a $5 \mathrm{~km}$. Encontró la existencia de múltiples rutas entre los parches de dos ecosistemas el matorral y bosque costero, con lo cual presentaron una alta concentración de flujo de especies. En este caso al utilizar una escala mayor, pudo identificar las concentraciones de movimiento tanto en la zona costera como en la cuenca alta.

En cualquiera de estos casos, la pérdida total de cuellos de botella puede implicar el aislamiento de las poblaciones, por lo que resultan indispensables para la conectividad del sistema, debido a que son sitios donde se generan cuellos de botella en el flujo a través del paisaje o funcionan como puentes para la conectividad (León et al., 2017).

Para la escala local utilizada en Las Lisas-La Barrona es crítico para la conectividad del ecosistema 
manglar identificar una zona de cuello de botella. Esto toma relevancia por el hecho de identificar rutas de conectividad a través del paisaje, ya que son criterios fundamentales para tomar las mejores decisiones durante intervenciones antropogénicas en el paisaje (Lee et al., 2014).

León y colaboradores (2017) también evaluaron zonas críticas o corredores, mediante el análisis de conectividad y de flujo de corriente acumulada, con lo cual identificaron zonas de cuello de botella en el municipio de Envigado, Antioquia en Colombia, evidenciando la limitada disponibilidad de rutas de movimiento a los cuellos de botella, y que estos segmentos constituyen prioridades de conservación, ya que la pérdida de una pequeña porción del segmento tendría un efecto sobre la conectividad (Martínez-Martí \& Sainz-Martínez, 2016).

Respecto a la conectividad del manglar de Las Lisas-La Barrona, fue posible identificar núcleos con alto aporte a la conectividad (La Barrona, El Jiote), parche puente o corredor como el canal de Chiquimulilla y un cuello de botella (segmento Las Lisas El ahumado), los cuales son elementos estratégicos para aplicar medidas de conservación, restauración y manejo. Si bien, un aspecto ideal a tener en cuenta sería el análisis a mayor escala, tanto espacial como temporal, el conocimiento de la conectividad del área generado por este estudio es básico y representa una base para ayudar a los esfuerzos de planificación marino costera de acuerdo a la importancia de estos elementos espaciales en términos de aporte a la conectividad y representando piezas fundamentales para las aves que utilizan los manglares como hábitat.

\section{Referencias}

Alonzo-F., A. M., Finegan, B., Brenes, C., Gunter, S., \& Palomeque, X. (2017). Evaluación de la conectividad estructural y funcional en el corredor de conservación Podocarpus-Yacuambi, Ecuador. Caldasia, 39(1), 143-156. doi:10.15446/ caldasia.v39n1.64324

Bartesaghi, M. L. (2015). Fragmentación y conectividad del paisaje costero para vertebrados e invertebrados prioritarios para la conservación (Tesis de maestría). Universidad de la República de Uruguay, Programa de Desarrollo de las Ciencias Básicas, Uruguay.

Bennet, A. F. (1998). Linkages in the Landscape: The role of corridors and connectivity in wildlife conservation. Gland, Suiza: International Union for Conservation of Nature.

Calabuig, E. (2013). Corredores, conectividad y ecología del paisaje. Dossier Ciudades, 1, 29-42.

Colorado, G. J., Vásquez, J. L., \& Mazo, I. N. (2017). Modelo de conectividad ecológica de fragmentos de bosque andino en Santa Elena (Medellín, Colombia). Acta Biológica Colombiana, 22(3), 379-393. doi:10.15446/abc.v22n3.63013

Consejo Nacional de Áreas Protegidas \& Programa de las Naciones Unidas para el Desarrollo. (2017). Estudio técnico del área de uso múltiple marino costera Las Lisas. Guatemala: Autor.

Cordero, D. G. (2014). Análisis de la conectividad para el mono carayá (Alouatta caraya) en un sistema silvopastoril del Chaco Húmedo sudamericano (Tesis de maestría). Universidad de Alcalá, Paraguay.

Costanza, R., d'Arge, R., Groot, R. de, Farberk, S., Grasso, M., Hannon, B., ... van den Belt, M. (1997). The value of the world's ecosystem services and natural capital. Nature, 387, 253260.

Dávila, V., García, M. J., \& López, A. (2014). Utilidad de la biodiversidad como indicador de sostenibilidad para la evaluación de la calidad ambiental de la costa Este del Pacifico de Guatemala (Inf-201320). Guatemala: Universidad de San Carlos de Guatemala Dirección General de Investigación y Centro de Estudios Conservacionistas.

Desrochers, A., Bélisle, M., Morand-Ferron, J., \& Bourque, J. (2011). Integrating GIS and homing experiments to study avian movement costs. Landscape Ecology, 26, 47-58. doi:10.1007/ s10980-010-9532-8

Eisermann, K., \& Avendaño, C. (2007). Áreas propuestas para la designación como IBA (Área importante para la conservación de aves) en Guatemala. Guatemala: Sociedad Guatemalteca de Ornitología y Bird Life International.

Environmental Systems Research Institute. (2016). ArCMap (Versión 10.6) [Software]. Estados Unidos: ESRI.

European Comission. (2018). Morphological Spatial Pattern Analysis. Recuperado de http://forest.jrc. ec.europa.eu/download/software/guidos/mspa/ 
European Space Agency. (2017). Satellite Image Sentinel 2. ESA Web Portal http://www.esa.int/ Our_Activities/Observing_the_Earth/Copernicus/ Sentinel-3

Gandini, M., \& Lara B. (2012). Análisis morfológico de la dinámica de fragmentos de Paspalum quadrifarium en una zona de la cuenca del río Salado del sur. Comisión de investigaciones Científicas de la provincia de Buenos Aires. Ponencia presentada en la $V$ Jornada y II Congreso Argentino de Ecología de Paisajes $A z u l$. Congreso llevado a cabo en Buenos Aires, Argentina.

Hernando, A., Velázquez, J., Rosario, R., Pérez, A., \& Grande, M. (2013). Medida de la fragmentación de los hábitats en la Red Natura como indicador de su estado de conservación. Ponencia presentada en el Sexto Congreso Forestal Español. Congreso llevado a cabo en Madrid, España.

Instituto de Investigación y Proyección sobre Ambiente Natural y Sociedad de la Universidad Rafael Landívar. (2018). Ecosistemas de Guatemala basado en el sistema de clasificación de zonas de vida. Guatemala: Autor.

International Union for Conservation of Nature. (2018). The IUCN Red List of Threatened Species. Version 2017-3. Recuperado de http://www. iucnredlist.org/

Lee, S. Y., Primavera, J. H., Dahdouh-Guebas, F., McKee, K., Bosire, J. O., Cannicci, S., ... Record, S. (2014). Ecological role and services of tropical mangrove ecosystems: a reassessment. Global Ecology and Biogeography, 23, 726-743. doi:10.1111/geb.12155

León, J. D., Sáenz, O. A., Ortiz, C. E., Cardona, D. M., Flórez, L. Z., Martínez, A, ... Cuervo, D. (2017). Estudio del valor histórico, cultural, paisajístico y evaluación de impactos del componente ambiental para la construcción del tramo 2b-Metroplús en escenarios con y sin proyecto, Municipio de Envigado, Antioquia. Medellín, Colombia: Universidad Nacional de Colombia, Facultad de Ciencias Agrarias.

MacDonald, M. A. (2003). The role of corridors in biodiversity conservation in production forest landscapes: A literature review forestry Tasmania. Tasforests, 14, 41 -52.
Martínez-Martí, J., \& Sainz-Martínez, J. (2016). Anillo verde de la bahía de Santander: Conectando la naturaleza y la ciudad. Evaluación de la conectividad ecológica en el área metropolitana de la bahía de Santander (Informe final proyecto LIFE14 NAT/ES/000699). Cantabria: Fundación Naturaleza Hombre.

McRae, B. H., Dickson, B. G., Keitt, T. H., \& Shah, V. B. (2008). Using circuit theory to model connectivity in ecology, evolution, and conservation. Ecology, 89(10), 27122724. doi:10.1890/07-1861.1

McRae, B. H., Shah, V.B., \& Mohapatra, T. K. (2013). Circuitscape 4 User Guide. The Nature Conservancy.

National Council for Air and Stream Improvement. (2004). NatureServe EO Specs: Separation Distance for Animals. Cary, NC: NatureServe

Olds, A., Pitt, K., Maxwell, P., \& Connolly, R. (2012). Synergistic effects of reserves and connectivity on ecological resilience. Australian Journal of Applied Ecology, 49, 1195-1203.

Pascual, L., \& Saura, S. (2008). Integración de la conectividad ecológica de los bosques en los instrumentos de planificación forestal a escala comarcal y regional. Revista Divulgación, 94, 31-37.

Pelletier, D., Clark, M., Anderson, M.G., Rayfield, B., Wulder, M. A., \& Cardille, J. A. (2014). Applying circuit theory for corridor expansion and management at regional scales: Tiling, pinch points, and omnidirectional connectivity. PLoS ONE, 9(1), 1-11. e84135. doi:10.1371/journal. pone. 0084135

Rose, A. (2013). Systematic comparison of two habitat connectivity modeling approaches: Least cost path and circuit theory. Recuperado de https:// tigerprints.clemson.edu/all_theses/1634

Salazar, C., \& Sigüenza, R. (2010). Modelos ecológicos conceptuales en humedales guatemaltecos como herramienta para evaluar los efectos potenciales de las actividades humanas sobre poblaciones de aves acuáticas y su hábitat (Fodecyt No. 062009). Guatemala: Secretaría Nacional de Ciencia y Tecnología. 
Saura, S., \& Rubio, L. (2010). A common currency for the different ways in which patches and links can contribute to habitat availability and connectivity in the landscape. Ecography 33, 523-537, 2010. doi:10.1111/j.1600-0587.2009.05760.x

Saura, S., \& Torné, J. (2009). Conefor Sensinode 2.2: A software package for quantifying the importance of habitat patches for landscape connectivity. Environmental Modelling \& Software, 24, 135 139.

Shanthala, B. S., Murthy, M. S., Debnath, B., \& Jha, C. S. (2016). Identification of potential habitat patches for connectivity using weighted linear combination (WLC) and integral index of Connectivity (IIC) at East Godavari District, Andhra Pradesh, India. Journal of the Indian Society of Remote Sensing, 4 (3), 385-394. doi:10.1007/s12524-015-0508-7.
Soille, P., \& Vogt, P. (2008). Morphological segmentation of binary patterns. Pattern Recognition Letters, 30, 4, 456-459. doi: 10.1016/j.patrec.2008.10.015

Tres, D., \& Reis, A. (12 al 22 de abril de 2007). La nucleación como propuesta para la restauración de la conectividad del paisaje. II Simposio Internacional sobre Restauración Ecológica, Cuba.

van den Belt, M., \& Cole, A. (2014). Ecosystem goods and services in marine protected areas (MPAs) (Science for Conservation, 326). New Zealand: Department of Conservation Te Papa Atawhai.

Vogt, P. \& Riitters, K. (2017). GuidosToolbox: Universal digital image object analysis. European Journal of Remote Sensing, 50(1), 352-361. do: $10.1080 / 22797254.2017 .1330650$ 